\title{
Time matters: pathological effects of repeated psychosocial stress during the active, but not inactive, phase of male mice
}

\author{
Manuela S Bartlang, Inga D Neumann ${ }^{1}$, David A Slattery', Nicole Uschold-Schmidt ${ }^{1}$, Dominik Kraus ${ }^{1}$, \\ Charlotte Helfrich-Förster and Stefan O Reber ${ }^{1}$
}

Department of Neurobiology and Genetics, University of Würzburg, 97074 Würzburg, Germany

${ }^{1}$ Department of Behavioural and Molecular Neurobiology, University of Regensburg, Universitätsstraße 31, 93053 Regensburg, Germany

(Correspondence should be addressed to S O Reber; Email: stefan.reber@biologie.uni-regensburg.de)

\begin{abstract}
Recent findings in rats indicated that the physiological consequences of repeated restraint stress are dependent on the time of day of stressor exposure. To investigate whether this is also true for clinically more relevant psychosocial stressors and whether repeated stressor exposure during the light phase or dark phase is more detrimental for an organism, we exposed male C57BL/6 mice to social defeat (SD) across 19 days either in the light phase between Zeitgeber time (ZT)1 and ZT3 (SDL mice) or in the dark phase between ZT13 and ZT15 (SDD mice). While SDL mice showed a prolonged increase in adrenal weight and an attenuated adrenal responsiveness to ACTH in vitro after stressor termination, SDD mice showed reduced dark phase home-cage activity on observation days 7 , 14 , and 20, flattening of the diurnal corticosterone rhythm, lack of social preference, and higher in vitro IFN $\gamma$ secretion
\end{abstract}

from mesenteric lymph node cells on day 20/21. Furthermore, the colitis-aggravating effect of SD was more pronounced in SDD than SDL mice following dextran sulfate sodium treatment. In conclusion, the present findings demonstrate that repeated SD effects on behavior, physiology, and immunology strongly depend on the time of day of stressor exposure. Whereas physiological parameters were more affected by SD during the light/inactive phase of mice, behavioral and immunological parameters were more affected by SD during the dark phase. Our results imply that repeated daily SD exposure has a more negative outcome when applied during the dark/active phase. By contrast, the minor physiological changes seen in SDL mice might represent beneficial adaptations preventing the formation of those maladaptive consequences.

Journal of Endocrinology (2012) 215, 425-437

\section{Introduction}

Chronic stress in humans is known to be a risk factor for many affective disorders including anxiety and depression (for reviews, see Herbert (1997), and Shalev (2009)) as well as somatic disorders such as inflammatory bowel disease (Duffy et al. (1991) and Levenstein et al. (2000), for review, see Reber (2011)). Moreover, several rodent studies have shown that chronic or repeated stressor exposure induces anxiety- (Reber et al. 2007, Slattery et al. 2011) and depressive-like behavior (Berton et al. 2006). However, the mechanisms behind stressrelated disorders or maladaptations are still far from being understood, due in part to a lack of adequate and translationally relevant stress paradigms (for review, see Reber (2011)). Chronic psychosocial stress paradigms represent the most promising approach in animal research to unravel the molecular basis for stress-promoted pathologies as they more accurately reflect the human situation (for review, see Zohar \& Westenberg (2000)). In support, and unlike physical stressors, the hypothalamo-pituitary-adrenal (HPA) axis responses to repeated psychosocial stressors, such as intermittent social defeat (SD) in rats, do not habituate (Tornatzky \& Miczek 1993). Intermittent SD refers to repeated exposure to a larger, dominant conspecific, which according to traditional definitions would seem to represent a repeated homotypic stressor. However, it is believed that repeated SD does not result in adaptation as it rather represents a heterotypic stressor for the defeated animal due to the behavioral differences displayed by the varying dominant conspecifics it is exposed to (Sgoifo et al. 2002).

Although the monoaminergic theories of depression have dominated scientific thinking over the past decades (for review, see Hirschfeld (2000)), newer theories have become more popular to explain the accumulating basic and clinical evidence (for review, see Slattery et al. (2004) and Katz et al. (2010)). One such theory states that a disturbed internal clock is causally involved in the development of depression (Winkler et al. (2005), for review, see Racagni et al. (2007)), which is supported by altered plasma cortisol rhythms (Deuschle et al. 1997) and sleep patterns observed in 
most depressed patients and the recent licensing of agomelatine, which re-synchronizes disrupted circadian rhythms (Yous et al. (1992), for review, see de Bodinat et al. (2010)). In line with this, it was recently shown in rodents that chronic unpredictable stress affects suprachiasmatic nucleus (SCN) functionality, the central pacemaker of the circadian system in mammals, by dampening the amplitude of circadian PERIOD2 (PER2) protein expression, one of the main clock proteins involved in internal rhythm generation (Jiang et al. 2011). In addition, various biological diurnal rhythms generated and fine-tuned by the SCN such as locomotor activity (Gorka et al. 1996), body temperature (Ushijima et al. 2006), heart rate (Grippo et al. 2003), sleep (Moreau et al. 1995), and plasma glucocorticoid (GC) levels (Ottenweller et al. 1994) are also affected by repeated exposure to non-psychosocial stressors.

Similar to GC levels, activity of the gut system is regulated in a circadian fashion and chronodisruption within the gastrointestinal tract was shown to result in several gastrointestinal diseases such as irritable bowel syndrome (for review, see Konturek et al. (2011)).

As peripheral organs express GC receptors, peripheral circadian rhythms like gastrointestinal oscillations can be shifted under stressful conditions (Balsalobre et al. 2000). Moreover, persistent stimulation of the HPA axis, e.g. by chronic stress is considered to be a crucial contributory factor to the incidence of spontaneous or aggravation of chemically induced colonic inflammation characterized by elevated interferon (IFN) $\gamma$ levels, reduced colon length, and an increased histological damage score (Reber et al. (2006), for review, see Reber (2011)).

With respect to the latter parameter, HPA axis activity and, thus, GC levels peak at the start of the activity phase, i.e. in the early morning in humans and early evening in most rodent species. Given that HPA axis activity/rhythmicity is controlled by SCN efferents to the paraventricular nucleus of the hypothalamus (for review, see Nader et al. (2010)), the key activator of the HPA axis during stress, it is likely that stressor exposures at distinct times of the light-dark cycle may generate differential acute stress responses. This, in turn, would lead to differential consequences of chronic/ intermittent stressor exposure, in terms of both outcome and severity, for an individual. In support, the few studies that have examined this hypothesis have shown that restraint stress has different effects on body weight development and adrenal gland weight, depending on the time of day of stressor exposure (Rybkin et al. (1997) and Perez-Cruz et al. (2009), for review, see Koolhaas et al. (2011)).

However, it is currently not known whether and to what extent a clinically more relevant chronic/intermittent psychosocial stressor affects biological rhythmicity and whether this is dependent on the time of day of stressor exposure. Therefore, the main aim of this study was to investigate whether selected behavioral, physiological, and immunological consequences of repeated SD exposure ( $2 \mathrm{~h} /$ day; 19 days) are dependent on the time of day of stressor exposure. For this purpose, male mice were exposed to SD either during the light (SDL) or dark (SDD) phase, and home-cage activity, social preference, anxiety-related behavior, basal plasma ACTH, and corticosterone (CORT) concentrations, as well as adrenal in vitro responsiveness to ACTH, IFN $\gamma$ secretion from mesenteric lymph node cells, and the severity of dextran sulfate sodium (DSS)-induced colitis were assessed at different times of the day.

\section{Materials and Methods}

\section{Animals}

Male C57BL/6 mice (Charles River, Sulzfeld, Germany) weighing 19-22 g (experimental mice) or 30-35 g (used as residents during the stress procedure) were individually housed in standard polycarbonate mouse cages $(16 \times 22 \times 14 \mathrm{~cm}$; experimental mice) or polycarbonate observation cages $(38 \times 22 \times 35 \mathrm{~cm}$; residents) for at least 1 week before the start of the experiment. All mice were kept under standard laboratory conditions $\left(22 \pm 2{ }^{\circ} \mathrm{C} ; 60 \pm 5 \%\right.$ humidity) and had free access to tap water and standard mouse diet. They were exposed to a $12 \mathrm{~h}$ light: $12 \mathrm{~h}$ darkness cycle (light phase: $200 \pm 50$ lux) with lights-on at $0700 \mathrm{~h}$. The time of lights-on is defined as Zeitgeber time (ZT)0 and lights-off as ZT12. All experimental protocols were approved by the Committee on Animal Health and Care of the local government and performed according to international guidelines on the ethical use of animals. All efforts were made to minimize the number of animals used and their sufferings.

\section{Experimental procedures}

After delivery, mice were weighed and randomly assigned to a single-housed control (SHC), a SDL, and a SDD group (Fig. 1). SHC mice remained undisturbed except for weighing on days 0 and 20 (experiment 1; Fig. 1A) and a weekly change of bedding (all experiments). Based on previous studies, SHC mice were considered to be the most appropriate controls in this paradigm (Singewald et al. 2009). SDL and SDD mice were exposed daily for $2 \mathrm{~h}$ to SD over 19 days (except for days 7 and 14; experiment 1,2, and 4) or over 4 days (experiment 3 ), either at the beginning of the light phase (ZT1-ZT3, SDL mice) or at the beginning of the dark phase (ZT13-ZT15, SDD mice) (see Fig. 1). The experimental design of the SDL and SDD procedure (for detailed description, see below) was identical for all experiments. The majority of parameters, except behavior (social preference/ avoidance test, SPAT; elevated plus-maze, EPM), were assessed in both the light and dark phase in order to determine whether repeated stressor exposure resulted in phase-dependent or -independent alterations.

Experiment 1 In order to assess whether submission latencies, the acute behavioral stress response (active behavior 
(A)

Experiment 1
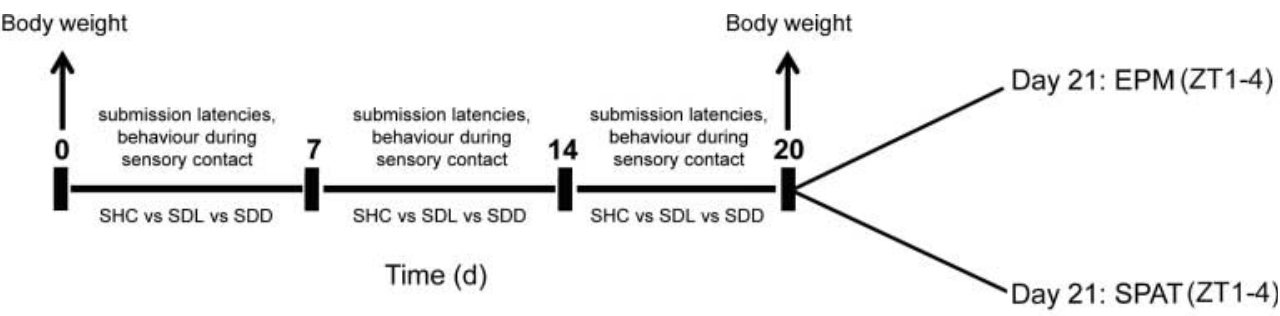

(B)

\section{Experiment 2}

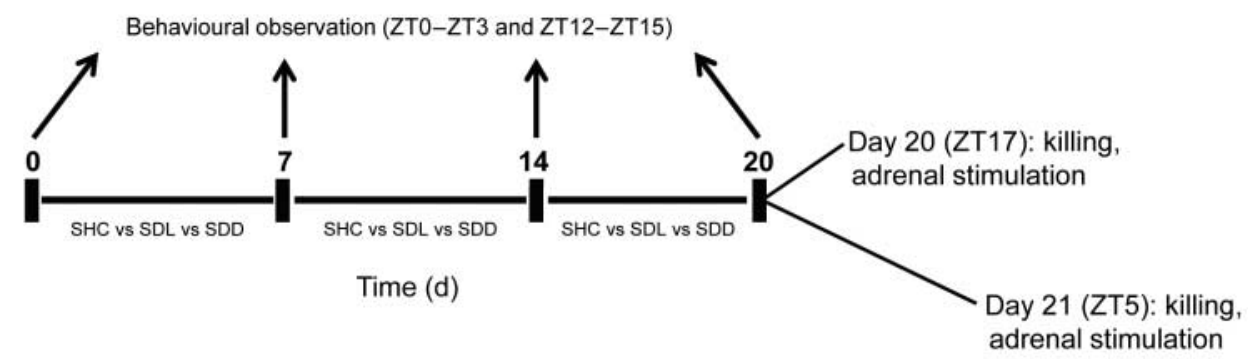

(C)

\section{Experiment 3}

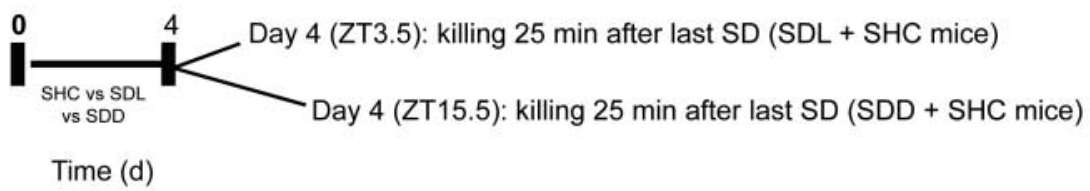

(D)
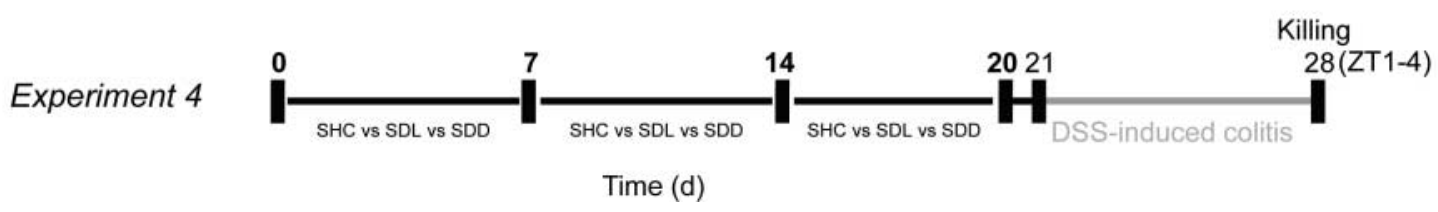

Time (d)

Figure 1 Schematic illustration of the experimental procedures. Description of the procedures performed in experiments 1-4 including their timeline. SHC, single-housed control; SDL, social defeat light; SDD, social defeat dark.

and time spent in contact zone during sensory contact), and social preference and/or anxiety are dependent on the time of day of stressor exposure, separate cohorts of animals were used for assessment of these parameters (Fig. 1A). In detail, submission latencies before and after $2 \mathrm{~h}$ of sensory contact and active behavior shown during sensory contact were assessed for SDL $(n=14)$ and SDD $(n=15)$ mice. Furthermore, SHC $(n=14)$, SDL $(n=14)$, and SDD $(n=14)$ mice were weighed before the start (day 0) and following termination of the SDL/SDD paradigm (day 20) to investigate whether the time of day of stressor exposure differentially affects body weight. Subsequently, respective mice were either exposed to the SPAT (SHC: $n=9$; SDL: $n=10$; SDD: $n=9$ ) or the EPM (SHC: $n=8$; SDL: $n=9$; SDD: $n=9$ ) at the beginning of the light phase (ZT1-ZT4) on day 21. We chose the light phase for these tests because preliminary data (confirmed by the results from experiment 2) showed that locomotion during the light phase was not different in SDL and SDD mice (data not shown), in contrast to locomotion during the dark phase (see results from experiment $1 \mathrm{~B})$.
Experiment 2 To assess whether the time of day of stressor exposure has an effect on active home-cage behavior, climbing, locomotion, rearing, eating, drinking, grooming, and digging were scored in another set of SHC $(n=24)$, SDL $(n=25)$, and SDD $(n=23)$ mice on days $0,7,14$, and 20 in the light phase from ZT0 to ZT3 and in the dark phase from ZT12 to ZT15 (Fig. 1B). In order to investigate whether the time of day of stressor exposure influences morning and/or evening basal plasma CORT and ACTH levels, adrenal gland weight, adrenal in vitro responsiveness to $\mathrm{ACTH}$, and anti-CD3-stimulated in vitro IFN $\gamma$ secretion from mesenteric lymph node cells, SHC, SDL, and SDD mice used for behavioral observations were subsequently killed by decapitation under brief inhalation anesthesia either in the dark phase (day 20, ZT17; $n=7-8$ per group) or in the light phase (day $21, \mathrm{ZT} 5 ; n=6-7$ per group), organs were removed and trunk blood was collected.

Experiment 3 Based on our findings from experiment 2, we assessed in a next step whether four daily sessions of repeated SD during either light (SDL) or dark (SDD) phase were sufficient to result in HPA axis habituation (Fig. 1C). 
Therefore, separate groups of SHC $(n=16)$, SDL $(n=8)$, and SDD $(n=8)$ mice were killed $25 \mathrm{~min}$ after the last defeat (SDL and respective SHC mice in the light phase at $\sim \mathrm{ZT} 3 \cdot 5$; SDD and respective SHC mice in the dark phase at $\sim$ ZT15.5) on day 4 of SD exposure. Trunk blood was collected for quantification of plasma ACTH levels.

Experiment 4 To assess whether the time of day of stressor exposure has an impact on the severity of a subsequent pharmacologically-induced colitis, SHC $(n=9)$, SDL $(n=10)$, and SDD $(n=9)$ mice were treated with $1 \%$ DSS in their drinking water for 8 days (Reber et al. 2008), starting on day 21 (day 21-28) (Fig. 1D). After killing on day 28, colon length was measured, the histological damage was scored, and IFN $\gamma$ secretion from mesenteric lymph node cells was determined.

\section{$S D L$ and $S D D$ stress paradigms}

During each SD session, the experimental mouse was taken from its home-cage and placed into a male resident's homecage. After the first attack by the resident that resulted in submissive behavior from the intruder, both mice were separated from each other by a perforated partition wall. This protected the intruder from physical injuries by the resident, but allowed visual, olfactory, and auditory contact as described previously (Reber et al. 2006). The submission latency of every intruder was assessed in order to make sure that the level of aggression received during $\mathrm{SD}$ is comparable for SDL and SDD mice. During the subsequent $2 \mathrm{~h}$ of sensory contact, food pellets, but not tap water, were available for all experimental mice including SHC mice. Furthermore, the time spent in the $3.5 \mathrm{~cm}$ broad contact zone (zone adjacent to the partition wall) and the active behavior (climbing, locomotion, rearing, eating, grooming, and digging) was assessed for the intruder every $3 \mathrm{~min}$ (note: values represent the sum of all active behaviors per intruder mouse). These 3-min scores were then summed up for 30-min periods per experimental mouse in each $2 \mathrm{~h}$ SD session, averaged per treatment group, and expressed in \% of total counts possible per 30 min interval, finally resulting in four 30 -min values per treatment group (0-30, 30-60, 60-90, and 90-120 min). Following removal of the partition wall, direct social interaction between the resident and the experimental mouse was allowed until the first attack by the resident that resulted in submissive behavior from the intruder. Again, submission latency of the intruder was scored before placing the experimental mouse back into its home-cage. Each experimental mouse was exposed to a different resident every day to avoid habituation. SD was omitted on days 7 and 14 in every experiment, and, during experiment 2, home-cage behavior of experimental mice was observed and scored at the beginning of the light phase (ZT0-ZT3) and dark phase (ZT12-ZT15) on days 0, 7,14 , and 20 respectively. The behavior was scored in 3-min time intervals, thereby distinguishing between locomotion, rearing, eating, drinking, grooming, digging, and climbing (active behavior) and inactive behavior. These 3-min scores were then summed up for the whole 180-min period per mouse and averaged per treatment group.

\section{Social preference/avoidance test}

SHC, SDL, and SDD mice were exposed to the SPAT on day 21 between ZT1 and ZT4 as described in detail before (Slattery et al. 2011). Briefly, the experimental mouse was placed into the SPAT box (length: $45 \mathrm{~cm}$; width: $27 \mathrm{~cm}$; height: $27 \mathrm{~cm}$; light intensity: 10-40 lux) for $30 \mathrm{~s}$ to habituate to the unfamiliar environment before a small empty wire mesh cage (length: $10 \mathrm{~cm}$; width: $6.5 \mathrm{~cm}$; height: $5 \mathrm{~cm}$; nonsocial trial) was introduced to the SPAT box for $150 \mathrm{~s}$. This initial period gives an indication of general anxiety (novel object exposure). Afterward, this empty cage was exchanged for an identical cage containing an unfamiliar male mouse (social trial) for another $150 \mathrm{~s}$. The total time spent in the $8 \mathrm{~cm}$ broad contact zone around the wire mesh cage was recorded using EthoVision XT (Version 5.0.216, Noldus Information Technology, Wageningen, The Netherlands) during both $150 \mathrm{~s}$ trials. The box and empty cage were cleaned thoroughly before each test.

\section{Elevated plus-maze}

SHC, SDL, and SDD mice were tested on the EPM on day 21 between ZT1 and ZT4 for 5 min as described previously (Reber et al. 2007). Briefly, the EPM consisted of two open $(6 \times 30 \mathrm{~cm})$ and two closed $(6 \times 30 \times 17 \mathrm{~cm})$ arms radiating from a central platform $(6 \times 6 \mathrm{~cm})$ to form a plus-shaped figure, which was elevated $60 \mathrm{~cm}$ above the floor. Each mouse was placed on the central platform facing a closed arm at the start of the trial and the maze was cleaned thoroughly before each test. The number of entries into the closed arms (as a measure of general activity) and the time spent on the open arms (as a measure of anxiety-related behavior) were recorded by means of a video/computer setup to allow calculation of the percentage of time spent on open arms of the maze.

\section{Quantification of plasma CORT and ACTH by ELISA}

SHC, SDL, and SDD mice of experiment 2 (day 20, ZT17, or day $21, \mathrm{ZT5}$ ) and experiment 3 (day 4 , SDL and respective SHC mice in the light phase $\sim \mathrm{ZT} 3 \cdot 5$; SDD and respective SHC mice in the dark phase at $\sim$ ZT15.5) were killed rapidly by decapitation following brief inhalation anesthesia. Approximately $500 \mu \mathrm{l}$ trunk blood was collected in EDTA-coated tubes on ice (Sarstedt, Nümbrecht, Germany), centrifuged at $4{ }^{\circ} \mathrm{C}(2000 \mathrm{~g}, 10 \mathrm{~min})$, and finally stored at $-20^{\circ} \mathrm{C}$ until assayed using a commercially available ELISA for CORT (analytical sensitivity $<1.631 \mathrm{nmol} / 1$, intra-assay and interassay coefficients of variation $\leq 6.35 \%$, IBL International, Hamburg, Germany) and ACTH (analytical sensitivity $0 \cdot 22 \mathrm{pg} / \mathrm{ml}$, intra-assay and interassay coefficients of variation $\leq 7 \cdot 1 \%$, IBL International). 


\section{Adrenal gland weight}

SHC, SDL, and SDD mice of experiment 2 were killed either on day 20 (ZT17) or on day 21 (ZT5). After removal, the adrenals were pruned from fat and weighed separately. Subsequently, adrenal weights were summed (sum of respective left and right adrenal weight) per mouse and expressed as absolute adrenal weight (mg).

\section{ACTH stimulation of adrenal explants in vitro}

Adrenal stimulation was performed as described previously (Uschold-Schmidt et al. 2012). Briefly, left and right adrenals were separately weighed and stored in ice-cold DMEM/F-12 (Life Technologies, Inc.) containing $0 \cdot 1 \%$ BSA until all mice were killed and adrenals removed. Afterward, each left and right adrenal gland was cut into two halves each containing cortical and medullary tissues. The halves were then weighed and pre-incubated in $200 \mu \mathrm{l}$ DMEM/F-12 for $4 \mathrm{~h}\left(37^{\circ} \mathrm{C}\right.$, $95 \% \mathrm{O}_{2}, 5 \% \mathrm{CO}_{2}$ ) before any further treatment. Culture medium was then replaced and each half of one adrenal was incubated with either medium containing $0.9 \%$ saline (basal) or medium containing $0.9 \%$ saline plus ACTH $(100 \mathrm{nM})$ for $6 \mathrm{~h}$ at $37{ }^{\circ} \mathrm{C}\left(95 \% \mathrm{O}_{2}\right.$ and $\left.5 \% \mathrm{CO}_{2}\right)$. Incubation of adrenals with either saline or ACTH took place on day 21 from ZT1 to ZT7 when mice were killed on day 20 (ZT17) and on day 21/22 from ZT13 to ZT19 when mice were killed on day 21 (ZT5). After incubation, supernatants were carefully removed and stored at $-20{ }^{\circ} \mathrm{C}$ until analyzed using a commercially available ELISA for CORT (IBL International). Basal as well as ACTH-induced CORT concentrations (ng/ml) in the supernatants were summed up afterwards for left and right adrenal per mouse respectively, and expressed in relation to respective adrenal explants' weight (sum of respective left and right adrenal explants) ( $\mathrm{ng} / \mathrm{ml}$ per $\mathrm{mg}$ ).

Secretion of IFN $\gamma$ from anti-CD3-stimulated mesenteric lymph node cells

Isolation and anti-CD3 stimulation of mesenteric lymph node cells from SHC, SDL, and SDD mice (experiment 2, day 20/21; experiment 4, day 28) were performed as described in detail before (Reber et al. 2006, 2008). Briefly, mesenteric lymph nodes (pooled from each experimental group) were harvested under sterile conditions and collected on ice in cell culture medium (RPMI 1640 supplemented with 10\% fetal bovine serum (PAA, Cölbe, Germany), $100 \mathrm{U} / \mathrm{ml}$ penicillin and $100 \mu \mathrm{g} / \mathrm{ml}$ streptomycin (PAA), and $3 \times 10^{-5}$ M 2-mercaptoethanol (Sigma)). Lymph nodes were mechanically disrupted and filtered through a cell strainer (70 $\mu \mathrm{m}$ Nylon, Falcon, Becton Dickinson, Heidelberg, Germany). Cells were washed twice in cell culture medium and adjusted to a concentration of $10^{6}$ cells $/ \mathrm{ml}$. Then, $2 \times 10^{5}(200 \mu \mathrm{l})$ lymph node cells were transferred to wells of a 96-well plate; to stimulate the cells, the wells were pre-coated with $100 \mu \mathrm{l}$ of $2.5 \mu \mathrm{g} / \mathrm{ml}$ anti-CD3 antibody.
Eight wells were loaded with the respective number of cells for each experimental group. After incubation for $24 \mathrm{~h}$ $\left(37{ }^{\circ} \mathrm{C}, 5 \% \mathrm{CO}_{2}\right)$, concentrations of the cytokine IFN $\gamma$ were measured in the supernatants by ELISA (Thermo Fisher Scientific, Rockford, IL, USA) according to the respective protocols, using four wells per experimental group.

\section{Determination of the severity of DSS-induced colitis}

Acute colitis was induced by administering 1\% DSS (36-50 kDa; ICN, Eschwege, Germany) in the drinking water ad libitum for 8 days as described previously (Reber et al. 2006, 2008), starting on day 21.

On day 28, inflammatory shortening of colonic length and the histological damage score were used to quantify the severity of the DSS-induced colonic inflammation as described previously (Reber et al. 2006, 2008). Briefly, the colon was removed, mechanically cleaned, and measured to $0.1 \mathrm{~cm}$ precision. Afterward, $1 \mathrm{~cm}$ of the distal third of the colon was cut longitudinally, laid on filter paper, and fixed in $10 \%$ formalin overnight. The next day, the fixed tissue was embedded in paraffin and cut longitudinally. Three $3 \mu \mathrm{m}$ hematoxylin-eosin-stained sections taken $100 \mu \mathrm{m}$ apart were evaluated by histological scoring performed by an investigator blind to treatment. For statistics, each individual score represented the mean of the three sections. Histology was scored as follows after (Obermeier et al. 2003, Reber et al. 2007, 2008): epithelium (0, normal morphology; 1 , loss of goblet cells; 2, loss of goblet cells in large areas; 3 , loss of crypts; 4 , loss of crypts in large areas) and infiltration (0, no infiltration; 1, infiltrate around crypt basis; 2, infiltrate reaching to lamina muscularis mucosae; 3 , extensive infiltration reaching the lamina muscularis mucosae and thickening of the mucosa with abundant edema; 4, infiltration of the lamina submucosa). The total histological score represents the sum of the epithelium and infiltration score and ranges from 0 to 8 .

\section{Statistical analysis}

For statistical comparisons, the software package SPSS (version 19) was used. One-way ANOVA (factor = treatment (SHC vs SDL vs SDD)) followed by Bonferroni post hoc test, if appropriate, was used for analysis of changes in body weight gain across SD, in the time spent on open arms and entries into closed arms during EPM testing, and in delta body weight gain, colon length, histological damage score, and IFN $\gamma$ secretion from mesenteric lymph node cells during DSS treatment. ANOVA for repeated measures (rm ANOVA) followed by Bonferroni post hoc test, if appropriate, was used for analysis of the time spent in the contact zone during the SPAT (factor $1=$ trial (empty cage vs mouse in cage) and factor $2=$ treatment (SHC vs SDL vs SDD)) and of the number of active behaviors during home-cage observations (factor $1=$ day $(0,7,14$, and 20) and factor $2=$ treatment (SHC vs SDL vs SDD)). All comparisons depending on two 


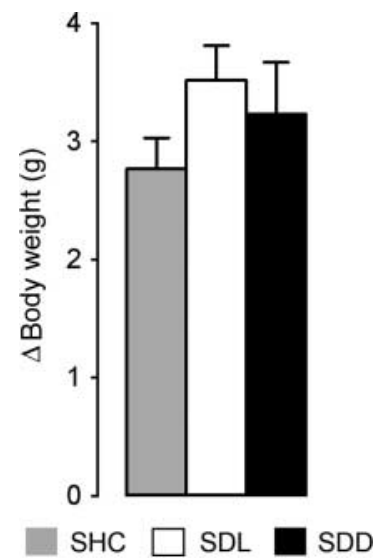

Figure 2 Effect of SD during light phase (SDL) and dark phase (SDD) on body weight. Body weight gain of SHC ( $n=14$, grey bars), SDL ( $n=14$, white bars), and SDD $(n=14$, black bars) mice across the stress procedure. Data represent mean weight gain (between day 0 and 20)+ S.E.M.

factors (absolute adrenal weight, basal plasma ACTH, and CORT levels, IFN $\gamma$ secretion from mesenteric lymph nodes (in mice not treated with DSS) (factor $1=$ treatment (SHC vs SDL vs SDD) and factor $2=$ time of day); in vitro adrenal CORT secretion (factor $1=$ treatment (SHC, SDL, and SDD) and factor $2=$ medium (ACTH vs saline)); submission latency (factor $1=$ treatment (SHC vs SDL vs SDD) and factor $2=$ interval (0-30 vs 30-60 vs 60-90 vs 90-120)) were performed using a two-way ANOVA followed by Bonferroni post hoc test if appropriate. Acute plasma ACTH responses to the fourth SD were analyzed separately for SDL and SDD mice (both vs respective SHC mice) by Mann-Whitney $U$ test. Data are presented as mean + S.E.M. Significance was set at $P \leq 0 \cdot 05$.

\section{Results}

Neither SDL nor SDD affects body weight development (experiment 1)

The body weight gain between days 0 and 20 was not different between SHC, SDL, and SDD mice (Fig. 2).

Submission latencies and behavioral patterns shown during SDL and SDD are comparable (experiment 1)

SDL and SDD mice did not differ in the time spent active nor in the time spent in the contact zone during $2 \mathrm{~h}$ of sensory contact. Furthermore, the submission latency before and after sensory contact was comparable between SDL and SDD mice (Table 1).

\section{SDD, but not SDL, decreases social preference (experiment 1)}

The absolute time spent in the contact zone during the SPAT was dependent on the stimulus (empty cage or mouse;
$\left.F_{1,25}=15 \cdot 9 ; P \leq 0 \cdot 001\right)$. Post hoc analysis showed that only SHC $(P \leq 0 \cdot 01)$ and SDL $(P \leq 0 \cdot 01)$, but not SDD, mice spent significantly more time in the social contact zone (mouse) compared with the nonsocial contact zone (empty cage) (Fig. 3A), thus showing social preference. The time spent exploring the empty cage was not different between the three groups.

Neither SDL nor SDD affects anxiety-like behavior (experiment 1)

In agreement with the SPAT results, neither the percentage of time spent in the open arms of the EPM, indicative of anxiety-like behavior, nor the number of closed arm entries, indicative of locomotor behavior, was statistically different between SHC, SDL, and SDD mice (Fig. 3B and C).

$S D D$, but not SDL, reduces dark phase activity on stress-free days 7, 14, and 20 (experiment 2)

Active behavior in the dark phase was found to be dependent on both the day of observation and the SD paradigm employed $\left(F_{2,69}=20 \cdot 9 ; P \leq 0 \cdot 001\right)$. Post hoc analysis showed that SDD mice were significantly less active on days 7,14 , and 20 compared with SHC $(P \leq 0 \cdot 001)$ and SDL $(P \leq 0 \cdot 001)$ mice and compared with respective day 0 values $(P \leq 0 \cdot 001$; Fig. 4). By contrast, active behavior in the light phase was neither dependent on the day of observation nor on the SD paradigm employed (data not shown).

\section{SDL and SDD increase adrenal gland weight (experiment 2)}

Absolute adrenal gland weight was dependent on the SD paradigm employed $\left(F_{2,38}=19 \cdot 4 ; P \leq 0 \cdot 001\right)$. Post hoc analysis revealed that, in comparison with SHC mice, SDL $(P \leq 0 \cdot 01)$

Table 1 Submission latency before and after sensory contact and analysis of behavior shown during sensory contact (experiment 1). Submission latencies and the acute behavioral stress response (active behavior and time spent in contact zone) of SDL $(n=14)$ and $\operatorname{SDD}(n=15)$ mice. Data represent mean \pm S.E.M.

$$
\text { SDL }(n=14) \quad \text { SDD }(n=15)
$$

Time spent in contact zone during sensory contact (\%)

$\begin{array}{lcc}0-30 \mathrm{~min} & 17 \pm 0 \cdot 013 & 20 \pm 0 \cdot 016 \\ 30-60 \mathrm{~min} & 18 \pm 0 \cdot 017 & 22 \pm 0 \cdot 021 \\ 60-90 \mathrm{~min} & 20 \pm 0 \cdot 018 & 20 \pm 0 \cdot 018 \\ 90-120 \mathrm{~min} & 19 \pm 0 \cdot 021 & 23 \pm 0 \cdot 016 \\ \text { Active behavior during sensory contact }(\%) & \\ 0-30 \mathrm{~min} & 82 \pm 0 \cdot 030 & 84 \pm 0 \cdot 023 \\ 30-60 \mathrm{~min} & 81 \pm 0 \cdot 029 & 80 \pm 0 \cdot 027 \\ 60-90 \mathrm{~min} & 79 \pm 0 \cdot 033 & 79 \pm 0 \cdot 025 \\ 90-120 \mathrm{~min} & 76 \pm 0 \cdot 037 & 79 \pm 0 \cdot 023 \\ \text { Submission latency }(\mathrm{s}) & & \\ \text { Before SD } & 76 \cdot 7 \pm 8 \cdot 55 & 66 \cdot 1 \pm 6 \cdot 96 \\ \text { After SD } & 74 \cdot 4 \pm 6 \cdot 10 & 64 \cdot 5 \pm 6 \cdot 02\end{array}$



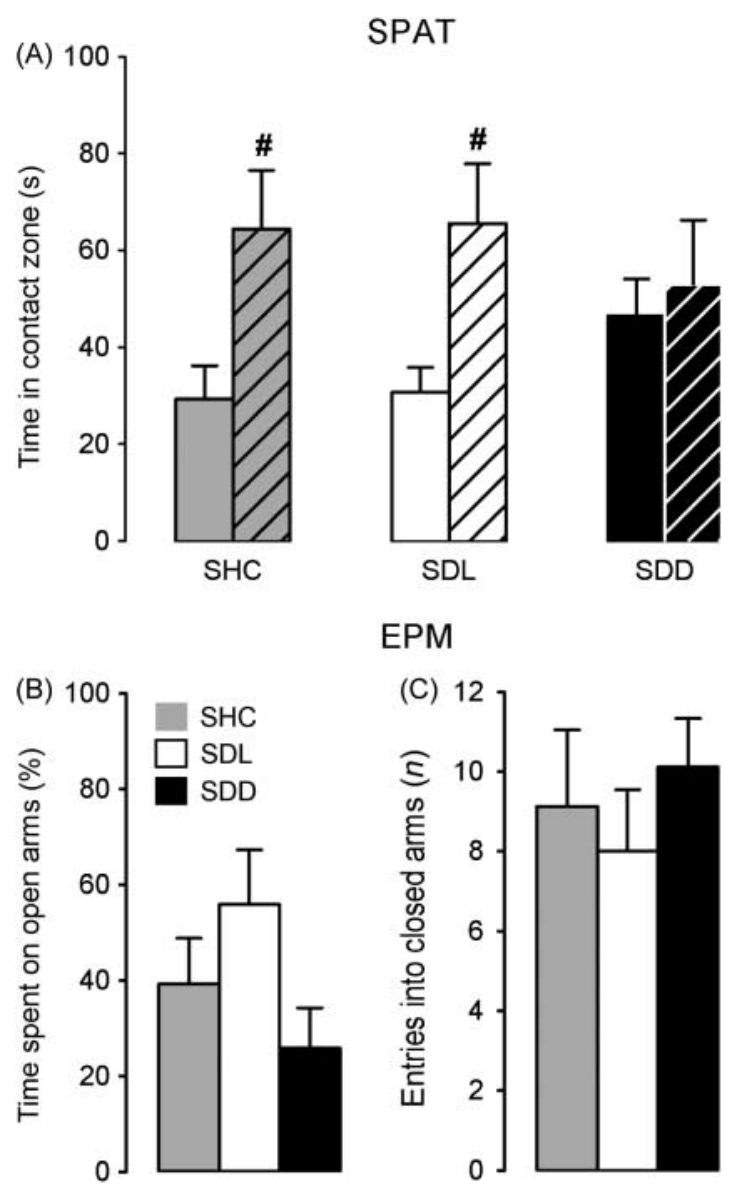

Figure 3 Effects of SD during the light phase (SDL) and dark phase (SDD) on social and general anxiety. To assess chronic psychosocial stress effects on social preference, SHC $(n=9), \operatorname{SDL}(n=10)$, and $\operatorname{SDD}(n=9)$ mice were exposed to the social preference/avoidance test (SPAT) on day 21 between ZT1 and ZT4 (A). Data show the absolute time (s) spent in the $8 \mathrm{~cm}$ broad contact zone around the wire mesh cage without (solid bars) and with (striped bars) a conspecific mouse present; with both trials lasting $150 \mathrm{~s}$ each. Data represent mean + S.E.M.; ${ }^{\#} P \leq 0.05$ vs respective group in the nonsocial trial. To assess anxiety-like behavior, $\operatorname{SHC}(n=8$, grey bars), $\operatorname{SDL}(n=9$, white bars), and SDD ( $n=9$, black bars) mice were exposed to the elevated plus-maze (EPM) on day 21 (ZT1-ZT4; $B$ and $C)$. Data show the $\%$ time on open arms (B) and the number of entries into closed arms (C). Data represent mean +S.E.M.

and $\operatorname{SDD}(P \leq 0 \cdot 01)$ mice adrenal gland weight was significantly increased when measured in the mid-dark phase. However, when measured in the mid-light phase, adrenal gland weight was significantly increased in SDL, but not SDD, mice $(P \leq 0 \cdot 001)$ in comparison with respective SHC mice (Fig. 5A).

SDD and SDL result in a loss of plasma ACTH rhythmicity (experiment 2)

Plasma ACTH was found to be affected by both the time of day of trunk blood collection and the SD paradigm employed

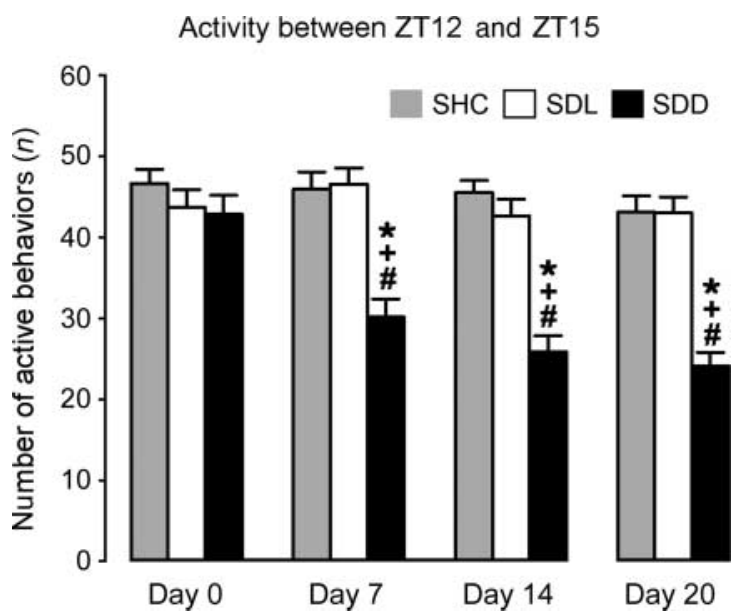

Figure 4 Effects of SD during light phase (SDL) and dark phase (SDD) on active behavior. To assess active home-cage behavior during the first $3 \mathrm{~h}$ following lights-off (ZT12-ZT15) on days 0,7 , 14 , and 20, climbing, locomotion, rearing, eating, drinking, grooming, and digging behaviors were scored in the home-cage of SHC ( $n=24$, grey bars), SDL ( $n=25$, white bars), and SDD $(n=23$, black bars) mice. Data represent mean + S.E.M.; ${ }^{*} P \leq 0.05$ vs respective $\mathrm{SHC}$ mice; ${ }^{+} P \leq 0.05$ vs respective $\mathrm{SDL}$ mice; ${ }^{*} P \leq 0.05$ vs respective group on day 0 . 
(A)

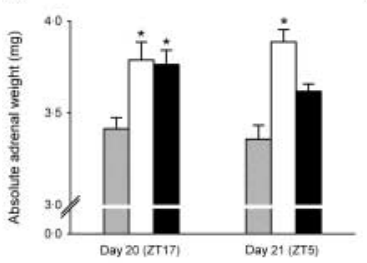

(D)

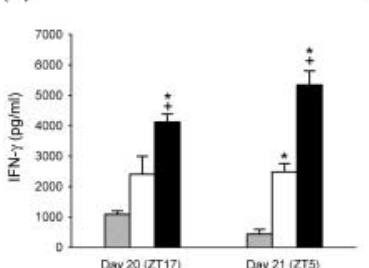

(B)

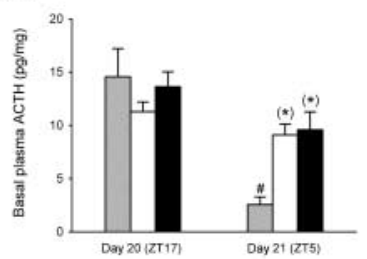

(E)

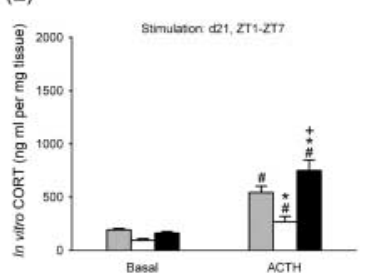

(C)

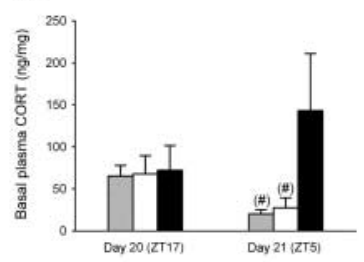

(F)

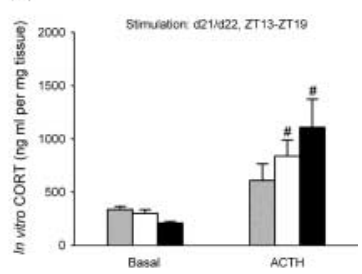

Figure 5 Effects of SD during the light phase (SDL) and dark phase (SDD) on adrenal weight, basal plasma ACTH and CORT, IFN $\gamma$, and adrenal in vitro ACTH responsiveness. Separate cohorts of SHC (grey bars), SDL (white bars), and SDD mice (black bars) were killed on day 20 (ZT17; $38 \mathrm{~h} / 26 \mathrm{~h}$ after the last SD for SDL/SDD mice; $n=7-8$ per group) or on day 21 (ZT5; $50 \mathrm{~h} / 38 \mathrm{~h}$ after the last SD for SDL/SDD mice; $n=6-7$ per group). Trunk blood was collected for quantification of basal plasma ACTH (B) and CORT (C). In each set the adrenal glands were removed, pruned from fat and weighed (A). Subsequently, adrenal explants were incubated with either medium containing saline (basal) or medium containing ACTH $(100 \mathrm{nM})$ for $6 \mathrm{~h}$ (day 21, ZT1-ZT7; day 21/day 22, ZT13-ZT19). Afterwards, CORT concentrations in the supernatants $(\mathrm{ng} / \mathrm{ml})$ were quantified and expressed per $\mathrm{mg}$ of adrenal tissue (ng/ml per $\mathrm{mg}$ ) (E and F). In addition, in vitro anti-CD3-stimulated IFN $\gamma$ secretion from mesenteric lymph node cells was assessed (D). Data represent mean + S.E.M.; ${ }^{*} P \leq 0 \cdot 05$ vs respective SHC mice; ${ }^{+} P \leq 0 \cdot 05$ vs respective SDL mice; ${ }^{\#} P \leq 0 \cdot 05$ vs respective group on day 20, ZT17 (B and C) or vs respective basal ( $\mathrm{E}$ and $\mathrm{F}$ ); ${ }^{(*)} P \leq 0 \cdot 05$ (one-way ANOVA) vs respective SHC mice; ${ }^{(\#)} P \leq 0.05$ (Mann-Whitney $U$ test) vs respective group on day 20 (ZT17).

significantly higher plasma ACTH levels than respective SHC mice $(P \leq 0 \cdot 01$; Fig. $6 \mathrm{~B})$. This effect was absent in SDL mice (Fig. 6A).

$S D L$, but not $S D D$, results in a reduction of adrenal in vitro ACTH responsiveness (experiment 2)

When adrenal explants were stimulated during the light phase, CORT secretion was found to be dependent on both the SD paradigm employed and the medium used (ACTH or saline) $\left(F_{2,40}=7 \cdot 19 ; P \leq 0 \cdot 01\right)$. Post hoc analyses revealed a significant increase in CORT secretion after in vitro $\mathrm{ACTH}$ stimulation in SHC $(P \leq 0 \cdot 001)$, SDL $(P \leq 0 \cdot 05)$, and SDD $(P \leq 0 \cdot 001)$ mice (Fig. 5E). However, this was significantly less pronounced in SDL compared with both SHC $(P \leq 0 \cdot 01)$ and SDD $(P \leq 0 \cdot 001)$ mice. By contrast, SDD mice showed a more pronounced ACTH-induced CORT secretion compared with SHC mice $(P \leq 0 \cdot 05)$.

When adrenal explants were stimulated during the dark phase, CORT secretion was dependent only on the medium used (ACTH or saline) $\left(F_{1,34}=26 \cdot 2 ; P \leq 0 \cdot 001\right)$. Post hoc analysis showed that ACTH-treated adrenal explants from SDL $(P \leq 0 \cdot 01)$ and SDD $(P \leq 0 \cdot 001)$ mice secreted significantly more CORT than respective saline-treated ones (Fig. 5F).
SDD results in a more pronounced elevation of IFN $\gamma$ secretion from mesenteric lymph node cells than SDL (experiment 2)

IFN $\gamma$ secretion from mesenteric lymph node cells was significantly dependent on the SD paradigm employed $\left(F_{2,16}=52 \cdot 1 ; P \leq 0 \cdot 001\right)$. When mice were killed during the dark phase, SDD mice showed a significantly higher IFN $\gamma$ secretion compared with both SHC $(P \leq 0 \cdot 001)$ and SDL $(P \leq 0 \cdot 05)$ mice. When mice were killed during the light phase, both SDL $(P \leq 0 \cdot 01)$ and $\operatorname{SDD}(P \leq 0 \cdot 001)$ mice showed a significantly higher IFN $\gamma$ secretion than SHC mice. Again, this was more pronounced in SDD mice $(P \leq 0 \cdot 001$ vs SDL) (Fig. 5D).

SDD causes a more severe aggravation of DSS colitis than SDL (experiment 4)

Body weight The body weight development between days 1 (i.e. day 21) and 8 (i.e. day 28) of DSS treatment was found to be dependent on the SD paradigm employed $\left(F_{2,25}=3 \cdot 97 ; \quad P \leq 0 \cdot 05\right)$. Post hoc tests revealed that SDD mice lost significantly more body weight than SHC mice $(P \leq 0 \cdot 05$; Fig. 7A).

Colon length The colon length following DSS treatment was dependent on the SD paradigm employed $\left(F_{2,25}=4 \cdot 31\right.$; 


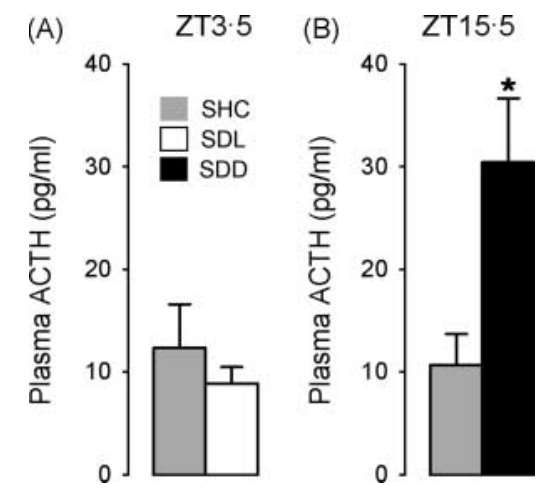

Figure 6 Effects of acute $\mathrm{SD}$ during the light phase $(\mathrm{SDL})$ and dark phase (SDD) on plasma ACTH. To assess the effect of four SD sessions on HPA axis activity, SHC, SDL, and SDD mice were killed 25 min after the last defeat on day 4 (A: SDL and SHC mice in the light phase at $\sim \mathrm{ZT} 3 \cdot 5, n=8$ per group; B: SDD and SHC mice in the dark phase at $\sim \mathrm{ZT} 15 \cdot 5 ; n=8$ per group) and plasma ACTH was measured. Data represent mean + S.E.M.; ${ }^{*} P \leq 0.05$ vs respective SHC mice.

$P \leq 0 \cdot 05)$. Post hoc analysis showed that SDD mice had a significantly shorter colon than SHC mice $(P \leq 0 \cdot 05$; Fig. 7B).

Histological damage score The histological damage score of the colon was dependent on the SD paradigm employed $\left(F_{2,25}=5 \cdot 96 ; P \leq 0 \cdot 01\right)$. Post hoc analysis revealed that both SDL and SDD mice showed a significantly increased histological damage score compared with SHC mice $(P \leq 0 \cdot 05$; Fig. 7C).

IFN $\gamma$ secretion from mesenteric lymph node cells IFN $\gamma$ secretion was dependent on the SD paradigm employed $\left(F_{2,9}=403 ; P \leq 0 \cdot 001\right)$. Post hoc analysis showed that IFN $\gamma$ secretion from mesenteric lymph node cells was significantly higher in SDD mice compared with both SHC $(P \leq 0 \cdot 001)$ and SDL $(P \leq 0 \cdot 001)$ mice (Fig. 7D).

\section{Discussion}

In this study, we demonstrate that the effects of chronic/ intermittent psychosocial stress on behavior, physiology, and immunology strongly depend on the time of day of stressor exposure, thus highlighting the importance of the circadian clock in this context. In more detail, while physiological parameters, such as absolute adrenal gland weight and adrenal ACTH responsiveness, were more affected by SD during the light phase, behavioral and immunological parameters like active home-cage behavior, social preference, IFN $\gamma$ secretion from mesenteric lymph node cells, and severity of DSS colitis were more affected by dark phase SD. Thus, our findings clearly show that the same stressor has a more pronounced negative outcome when applied during the active phase of an organism and provide the first evidence, suggesting that the physiological changes that occur in response to intermittent stressor exposure can be beneficial (SDL) or maladaptive (SDD).

Though there have been studies showing decreased body weight gain following repeated/chronic stressor exposure (Sachser \& Lick 1989, Berton et al. 1998), body weight in this study was not affected by SD, regardless of the time of day of stressor exposure. However, recent studies (Razzoli et al. 2011, Savignac et al. 2011, Slattery et al. 2011) have suggested that this parameter is not a reliable indicator of chronic stressor exposure.

In addition to body weight, assessment of adrenal gland weight and alterations in HPA axis activity are often used as hallmark signs of chronic stress. In line with the literature (Zelena et al. 2003, Reber et al. 2007), adrenal gland weight was increased in SDL and SDD mice in the mid-dark phase 1 day after stressor termination. However, this effect was only transient in SDD mice, as it disappeared $12 \mathrm{~h}$ later at a time after stressor termination where they were still enlarged in SDL mice. Support for a more pronounced effect of
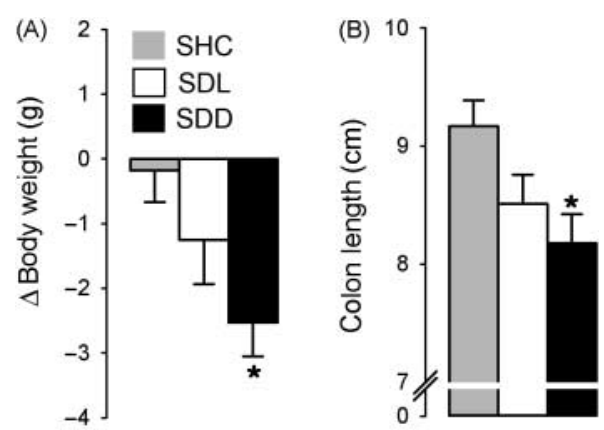

(C)

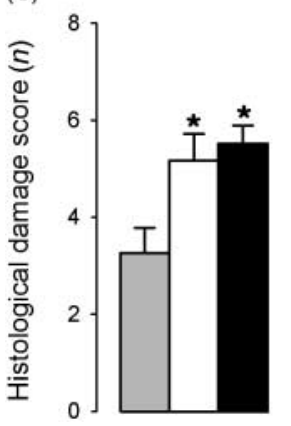

(D)

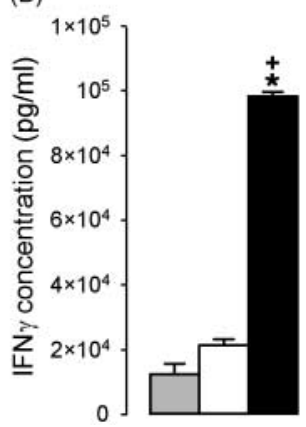

Figure 7 Effects of SD during the light phase (SDL) and dark phase (SDD) on the severity of DSS-induced colitis. To assess the consequences of SD on a chemically-induced colitis, SHC (grey bars, $n=9$ ), SDL (white bars, $n=10$ ), and SDD mice (black bars, $n=9$ ) were treated with $1 \%$ DSS in the drinking water from day 21 to day 28 in order to induce acute colitis. Body weight gain (A), colonic length (B), histological damage score of the colon (C) and IFN $\gamma$ secretion from anti-CD3-stimulated mesenteric lymph node cells $(D$, pooled per group and measured in quadruplets) were quantified to assess colitis severity. Data represent mean + S.E.M.; ${ }^{*} P \leq 0.05$ vs respective $\mathrm{SHC}$ mice; ${ }^{+} P<0.05$ vs respective SDL mice. 
light phase compared with dark phase stressor exposure on the HPA axis also comes from a previous study showing a higher acute HPA axis response, indicated by higher plasma ACTH and CORT levels, to restraint stress in the former phase (Kant et al. 1986).

Given these discrepancies in adrenal weight, we next assessed the influence of SDL and SDD on plasma CORT and ACTH. Interestingly, basal plasma CORT concentrations were not affected in either phase by repeated SD, regardless of the time of day of repeated stressor exposure. This finding is contradictory to studies reporting either hypercorticism (for review, see Bartolomucci et al. (2005), Ushijima et al. (2006)) or hypocorticism (Sanchez et al. 1998, Reber et al. 2006, 2007) as a consequence of chronic/intermittent psychosocial stressor exposure. However, basal plasma CORT levels in the dark phase were significantly higher than during the light phase in SHC and SDL, but not SDD, mice indicating a disturbed diurnal plasma CORT rhythm following dark phase stressor exposure. This lack, or shift, of CORT rhythmicity exclusively in SDD mice is of special relevance, given that both SDL and SDD mice showed disturbed diurnal plasma ACTH patterns (elevated basal light phase levels), when compared with SHC mice. Together, these findings strongly suggest that adrenals of SDL, but not those of SDD, mice develop an adaptive reduction in at least light phase ACTH responsiveness in order to maintain low basal light phase plasma GC levels and, thus, a vital diurnal CORT rhythm. In contrast to the transient increase in SDD mice, the more persistent increase in adrenal mass seen in SDL mice might be crucial for maintaining this vital CORT rhythm by exhibiting a reduced ACTH sensitivity during the light phase.

As other factors can influence CORT release in vivo, we next assessed the effects of ACTH on CORT secretion of adrenal explants in vitro. Our in vivo hypotheses are supported by our in vitro data assessing adrenal gland ACTH responsivity. While adrenal explants of all three treatment groups (SHC, SDL, and SDD) responded to ACTH with a significant increase in CORT secretion, this effect was blunted in SDL when adrenals were stimulated during the light phase on day 21. Intriguingly, the response of the SDD was even greater than that of the SHC mice at this timepoint, which supports our claim that these mice do not generate an adaptive ACTH insensitivity. Importantly, peripheral organs such as the adrenal glands show their intrinsic rhythm of clock gene expression (likely a measure of the rhythmicity of the tissue) for at least a few days after they had been removed from an animal, indicating that this process is not centrally regulated by the SCN (Yamazaki et al. 2000, 2009). Thus, the CORT secretion of adrenal explants during in vitro ACTH stimulation does not reflect the potential of the adrenal to secret CORT at the moment of killing, but at the actual stimulation period.

In addition to maintaining low light phase plasma CORT levels following stressor termination, the reduction in light phase adrenal ACTH sensitivity allows SDL mice to adapt their HPA axis responses to the temporally predictable repeated SD sessions taking place from ZT1 to ZT3 over 19 consecutive days (except observation days). Besides the decrease in light phase adrenal ACTH sensitivity, HPA axis adaptations in SDL mice are further seen at the level of the pituitary gland on day 4 of SDL. In detail, plasma ACTH was not affected in SDL, but increased in SDD mice, 25 min after termination of the SD session on day 4 of repeated stressor exposure, further indicating that the process of adaptation in SDL mice develops reasonably early in the context of repeated stressor exposure. Herefore, the decrease in adrenal ACTH sensitivity in SDL mice during the light phase seems more likely to represent an adaptational process to this predictable SD stressor than a maladaptive consequence of chronic/repeated SD.

In addition to the physiological and neuroendocrine changes that were found to strongly depend on the time of day of the stressor exposure, home-cage behavioral analyses revealed profound differences in the dark, but not light, phase activity profiles of the different treatment groups. SDD, but not SDL, mice showed a significant decrease in dark phase activity on days 7,14 , and 20 when compared with SHC mice. At least for the SDD group, our data are consistent with a previous study, reporting a decrease in activity during the active, but not inactive, phase after chronic mild stress in rats (Gorka et al. 1996). Given that SDD mice were predictably exposed to SD for 19 consecutive days (except observation days) at the beginning of the dark phase, a reduction in dark phase activity is likely to indicate that SDD mice are expecting the next SD. As a consequence, they show less motivation and drive during that phase, which are approved hallmarks of affective disorders such as depression and anxiety (Willner 1990, Liebsch et al. 1998). The finding that these behavioral alterations were lacking in SDL mice gives further support for our hypothesis that these mice actively develop coping mechanisms, which enable them to adapt better to the situation than SDD mice.

Further support for this is shown by the fact that SDL, but not SDD, mice display normal social preference toward an unfamiliar male conspecific. Social avoidance has also been described following 10 days of repeated SD with 24-h sensory contact (Berton et al. 2006, Krishnan et al. 2007) and was associated with depressive-like and anxiety-related behavioral abnormalities and, thus, can be interpreted as a maladaptive behavioral consequence (Lagace et al. 2010, Slattery et al. 2011).

Importantly, and in agreement with the EPM results of this study, the time spent in the nonsocial contact zone was not affected by SDL or SDD, suggesting that irrespective of the time of exposure, anxiety-like behavior is not influenced by repeated SD. In support, lack of behavioral signs of anxiety has also been found following 19 days of repeated SD and/or overcrowding (Slattery et al. 2011), which is in contrast with elevated levels of anxiety as a consequence of chronic psychosocial stress paradigms such as chronic subordinate colony housing (Reber et al. 2007, Slattery et al. 2011). 
Thus, these different social stress models have disparate behavioral and physiological effects akin to those seen following SDL and SDD respectively. Despite discrepancies in the above mentioned parameters, SD and/or overcrowding as well as chronic subordinate colony housing lead to the same immunological effects and aggravate DSS-induced colitis (Reber et al. 2006, 2008). Therefore, as the physiological and behavioral results of this study do not allow for a clear conclusion on whether they are adaptive or maladaptive, we investigated the immunological response to DSS-induced colitis, a model that is used to determine the maladaptive consequence of chronic psychosocial stress (Reber et al. (2006), for review, see Reber (2011)). Thus, SHC, SDL, and SDD mice were treated with DSS in their drinking water for 8 days subsequent to 19 days of SD. As expected, the severity of DSS-induced colitis was increased in SDL and SDD mice compared with SHC mice. In agreement with our and other previous data (Reber et al. (2007), for review, see Reber (2011), Savignac et al. (2011)), repeated/chronic psychosocial stress, induced by SD either during light or dark phase in this study, resulted in development of a mild spontaneous colonic inflammation, indicated by an increased histological damage score in both SDL and SDD mice. However, the inflammatory response was significantly more pronounced in SDD mice, indicated by a greater body weight loss, inflammatory reduction of colon length, and IFN $\gamma$ secretion from anti CD3-stimulated mesenteric lymph node cells in SDD compared with SDL mice. This strongly suggests that SD during the dark phase has a stronger pro-inflammatory potential compared with SD during the light phase. Thus, these findings further suggest both maladaptive affective and somatic consequences of repeated SD during the dark (active) phase, in contrast to SDL, which results in beneficial adaptations preventing these maladaptive effects.

Importantly, SDL and SDD mice did not differ in submission latencies toward their respective residents, as well as in time spent in the contact zone and time spent active during the $2 \mathrm{~h}$ of sensory contact. Therefore, it is very unlikely that any of the above described behavioral, physiological, and immunological differences between SDL and SDD mice are simply due to different levels of aggression received during the light phase (inactive period) and dark phase (active period) SD.

\section{General conclusion}

Overall, our study is the first to demonstrate that chronic/intermittent psychosocial stress effects on behavior, physiology, and immunology strongly depend on the time of day of stressor exposure. While physiological parameters such as adrenal gland weight and adrenal ACTH responsiveness were more affected by SD during the light phase, behavioral and immunological parameters like active home-cage behavior during the dark phase, social preference, IFN $\gamma$ secretion from mesenteric lymph node cells, and severity of a DSS-induced colitis were more affected by SD during the dark phase. In contrast to the physiological changes seen in SDL mice that are likely to represent beneficial adaptations, decreased interest in an unfamiliar conspecific, loss of general activity, a flattened diurnal CORT rhythm, and shift toward a more pro-inflammatory body milieu, occurring particularly in SDD mice, represent well-accepted hallmarks of affective disorders such as depression, and, thus, are considered to be maladaptive. Taken together, though further work is required to provide additional insight, our data suggest that equally severe stressors have a more pronounced negative outcome when applied during the active/dark phase. Moreover, they also suggest that the physiological changes seen in mice stressed during the rest period (SDL mice) might represent beneficial adaptations rather than maladaptive consequences.

\section{Declaration of interest}

The authors declare that there is no conflict of interest that could be perceived as prejudicing the impartiality of the research reported.

\section{Funding}

This study was supported by the Deutsche Forschungsgemeinschaft (DFG FO-207/13-1). The funders had no role in study design, data collection and analysis, decision to publish, or preparation of the manuscript.

\section{Author contribution statement}

M S B, I D N, D A S, C H-F, and S O R involved in the conception and design of the experiments; M S B, N U-S, D K, and S O R involved in the collection, analysis, and interpretation of data; M S B, I D N, D A S, C H-F, and S O R involved in drafting the article or revising it critically for important intellectual content.

\section{Acknowledgements}

The authors are grateful to N Grunwald, A Havasi, S Peters, A Füchsl, and K Altmann for their excellent technical help and to Dr I Karatsoreos for his helpful comments regarding the manuscript.

\section{References}

Balsalobre A, Brown SA, Marcacci L, Tronche F, Kellendonk C, Reichardt HM, Schutz G \& Schibler U 2000 Resetting of circadian time in peripheral tissues by glucocorticoid signaling. Science $\mathbf{2 8 9}$ 2344-2347. (doi:10.1126/science.289.5488.2344)

Bartolomucci A, Palanza P, Sacerdote P, Panerai AE, Sgoifo A, Dantzer R \& Parmigiani S 2005 Social factors and individual vulnerability to chronic stress exposure. Neuroscience \& Biobehavioral Reviews 29 67-81. (doi:10.1016/j.neubiorev.2004.06.009)

Berton O, Aguerre S, Sarrieau A, Mormede P \& Chaouloff F 1998 Differential effects of social stress on central serotonergic activity and emotional reactivity in Lewis and spontaneously hypertensive rats. Neuroscience 82 147-159. (doi:10.1016/S0306-4522(97)00282-0) 
Berton O, McClung CA, Dileone RJ, Krishnan V, Renthal W, Russo SJ, Graham D, Tsankova NM, Bolanos CA, Rios M et al. 2006 Essential role of BDNF in the mesolimbic dopamine pathway in social defeat stress. Science 311 864-868. (doi:10.1126/science.1120972)

de Bodinat C, Guardiola-Lemaitre B, Mocaer E, Renard P, Munoz C \& Millan MJ 2010 Agomelatine, the first melatonergic antidepressant: discovery, characterization and development. Nature Reviews. Drug Discovery 9 628-642. (doi:10.1038/nrd3274)

Deuschle M, Schweiger U, Weber B, Gotthardt U, Korner A, Schmider J, Standhardt H, Lammers CH \& Heuser I 1997 Diurnal activity and pulsatility of the hypothalamus-pituitary-adrenal system in male depressed patients and healthy controls. Journal of Clinical Endocrinology and Metabolism 82 234-238. (doi:10.1210/jc.82.1.234)

Duffy LC, Zielezny MA, Marshall JR, Byers TE, Weiser MM, Phillips JF, Calkins BM, Ogra PL \& Graham S 1991 Relevance of major stress events as an indicator of disease activity prevalence in inflammatory bowel disease. Behavioral Medicine 17 101-110. (doi:10.1080/08964289.1991. 9937553)

Gorka Z, Moryl E \& Papp M 1996 Effect of chronic mild stress on circadian rhythms in the locomotor activity in rats. Pharmacology, Biochemistry and Behavior 54 229-234. (doi:10.1016/0091-3057(95)02173-6)

Grippo AJ, Beltz TG \& Johnson AK 2003 Behavioral and cardiovascular changes in the chronic mild stress model of depression. Physiology \& Behavior 78 703-710. (doi:10.1016/S0031-9384(03)00050-7)

Herbert J 1997 Fortnighly review, Stress, the brain, and mental illness. BMJ 315 530-535. (doi:10.1136/bmj.315.7107.530)

Hirschfeld RM 2000 History and evolution of the monoamine hypothesis of depression. Journal of Clinical Psychiatry 61 (Suppl 6) 4-6.

Jiang WG, Li SX, Zhou SJ, Sun Y, Shi J \& Lu L 2011 Chronic unpredictable stress induces a reversible change of PER2 rhythm in the suprachiasmatic nucleus. Brain Research 1399 25-32. (doi:10.1016/j.brainres.2011.05.001)

Kant GJ, Mougey EH \& Meyerhoff JL 1986 Diurnal variation in neuroendocrine response to stress in rats: plasma $\mathrm{ACTH}, \beta$-endorphin, $\beta$-LPH, corticosterone, prolactin and pituitary cyclic AMP responses. Neuroendocrinology 43 383-390. (doi:10.1159/000124553)

Katz MM, Bowden CL \& Frazer A 2010 Rethinking depression and the actions of antidepressants: uncovering the links between the neural and behavioral elements. Journal of Affective Disorders 120 16-23. (doi:10.1016/j. jad.2009.08.011)

Konturek PC, Brzozowski T \& Konturek SJ 2011 Gut clock: implication of circadian rhythms in the gastrointestinal tract. Journal of Physiology and Pharmacology 62 139-150.

Koolhaas JM, Bartolomucci A, Buwalda B, de Boer SF, Flugge G, Korte SM, Meerlo P, Murison R, Olivier B, Palanza P et al. 2011 Stress revisited: a critical evaluation of the stress concept. Neuroscience \& Biobehavioral Reviews 35 1291-1301. (doi:10.1016/j.neubiorev.2011.02.003)

Krishnan V, Han MH, Graham DL, Berton O, Renthal W, Russo SJ, Laplant Q, Graham A, Lutter M, Lagace DC et al. 2007 Molecular adaptations underlying susceptibility and resistance to social defeat in brain reward regions. Cell 131 391-404. (doi:10.1016/j.cell.2007.09.018)

Lagace DC, Donovan MH, DeCarolis NA, Farnbauch LA, Malhotra S, Berton O, Nestler EJ, Krishnan V \& Eisch AJ 2010 Adult hippocampal neurogenesis is functionally important for stress-induced social avoidance. PNAS 107 4436-4441. (doi:10.1073/pnas.0910072107)

Levenstein S, Prantera C, Varvo V, Scribano ML, Andreoli A, Luzi C, Arca M, Berto E, Milite G \& Marcheggiano A 2000 Stress and exacerbation in ulcerative colitis: a prospective study of patients enrolled in remission. American Journal of Gastroenterology 95 1213-1220. (doi:10.1111/j.15720241.2000.02012.x)

Liebsch G, Montkowski A, Holsboer F \& Landgraf R 1998 Behavioural profiles of two Wistar rat lines selectively bred for high or low anxietyrelated behaviour. Behavioural Brain Research 94 301-310. (doi:10.1016/ S0166-4328(97)00198-8)

Moreau JL, Scherschlicht R, Jenck F \& Martin JR 1995 Chronic mild stressinduced anhedonia model of depression; sleep abnormalities and curative effects of electroshock treatment. Behavioural Pharmacology 6 682-687. (doi:10.1097/00008877-199511000-00003)
Nader N, Chrousos GP \& Kino T 2010 Interactions of the circadian CLOCK system and the HPA axis. Trends in Endocrinology and Metabolism 21 277-286. (doi:10.1016/j.tem.2009.12.011)

Obermeier F, Dunger N, Strauch UG, Grunwald N, Herfarth H, Scholmerich J \& Falk W 2003 Contrasting activity of cytosin-guanosin dinucleotide oligonucleotides in mice with experimental colitis. Clinical and Experimental Immunology 134 217-224. (doi:10.1046/j.1365-2249.2003.02288.x)

Ottenweller JE, Servatius RJ \& Natelson BH 1994 Repeated stress persistently elevates morning, but not evening, plasma corticosterone levels in male rats. Physiology \& Behavior 55 337-340. (doi:10.1016/0031-9384(94)90143-0)

Perez-Cruz C, Simon M, Flugge G, Fuchs E \& Czeh B 2009 Diurnal rhythm and stress regulate dendritic architecture and spine density of pyramidal neurons in the rat infralimbic cortex. Behavioural Brain Research $\mathbf{2 0 5}$ 406-413. (doi:10.1016/j.bbr.2009.07.021)

Racagni G, Riva MA \& Popoli M 2007 The interaction between the internal clock and antidepressant efficacy. International Clinical Psychopharmacology 22 (Suppl 2) S9-S14. (doi:10.1097/01.yic.0000277957.75852.c7)

Razzoli M, Carboni L, Andreoli M, Ballottari A \& Arban R 2011 Different susceptibility to social defeat stress of BalbC and C57BL6/J mice. Behavioural Brain Research 216 100-108. (doi:10.1016/j.bbr.2010.07.014)

Reber SO 2011 Stress and animal models of inflammatory bowel disease - an update on the role of the hypothalamo-pituitary-adrenal axis. Psychoneuroendocrinology 37 1-19. (doi:10.1016/j.psyneuen.2011.05.014)

Reber SO, Obermeier F, Straub RH, Falk W \& Neumann ID 2006 Chronic intermittent psychosocial stress (social defeat/overcrowding) in mice increases the severity of an acute DSS-induced colitis and impairs regeneration. Endocrinology 147 4968-4976. (doi:10.1210/en.2006-0347)

Reber SO, Birkeneder L, Veenema AH, Obermeier F, Falk W, Straub RH \& Neumann ID 2007 Adrenal insufficiency and colonic inflammation after a novel chronic psycho-social stress paradigm in mice: implications and mechanisms. Endocrinology 148 670-682. (doi:10.1210/en.2006-0983)

Reber SO, Obermeier F, Straub RH, Veenema AH \& Neumann ID 2008 Aggravation of DSS-induced colitis after chronic subordinate colony (CSC) housing is partially mediated by adrenal mechanisms. Stress 11 225-234. (doi:10.1080/10253890701733351)

Rybkin II, Zhou Y, Volaufova J, Smagin GN, Ryan DH \& Harris RB 1997 Effect of restraint stress on food intake and body weight is determined by time of day. American Journal of Physiology 273 R1612-R1622.

Sachser N \& Lick C 1989 Social stress in guinea pigs. Physiology \& Behavior 46 137-144. (doi:10.1016/0031-9384(89)90246-1)

Sanchez MM, Aguado F, Sanchez-Toscano F \& Saphier D 1998 Neuroendocrine and immunocytochemical demonstrations of decreased hypothalamo-pituitary-adrenal axis responsiveness to restraint stress after long-term social isolation. Endocrinology 139 579-587. (doi:10.1210/en. 139.2.579)

Savignac HM, Hyland NP, Dinan TG \& Cryan JF 2011 The effects of repeated social interaction stress on behavioural and physiological parameters in a stress-sensitive mouse strain. Behavioural Brain Research 216 576-584. (doi:10.1016/j.bbr.2010.08.049)

Sgoifo A, Pozzato C, Meerlo P, Costoli T, Manghi M, Stilli D, Olivetti G \& Musso E 2002 Intermittent exposure to social defeat and open-field test in rats: acute and long-term effects on ECG, body temperature and physical activity. Stress 5 23-35. (doi:10.1080/102538902900012387)

Shalev AY 2009 Posttraumatic stress disorder and stress-related disorders. Psychiatric Clinics of North America 32 687-704. (doi:10.1016/j.psc. 2009.06.001)

Singewald GM, Nguyen NK, Neumann ID, Singewald N \& Reber SO 2009 Effect of chronic psychosocial stress-induced by subordinate colony (CSC) housing on brain neuronal activity patterns in mice. Stress 12 58-69. (doi:10.1080/10253890802042082)

Slattery DA, Hudson AL \& Nutt DJ 2004 Invited review: the evolution of antidepressant mechanisms. Fundamental \& Clinical Pharmacology 18 1-21. (doi:10.1111/j.1472-8206.2004.00195.x)

Slattery DA, Uschold N, Magoni M, Bar J, Popoli M, Neumann ID \& Reber SO 2011 Behavioural consequences of two chronic psychosocial stress paradigms: anxiety without depression. Psychoneuroendocrinology 37 702-714. (doi:10.1016/j.psyneuen.2011.09.002) 
Tornatzky W \& Miczek KA 1993 Long-term impairment of autonomic circadian rhythms after brief intermittent social stress. Physiology \& Behavior 53 983-993. (doi:10.1016/0031-9384(93)90278-N)

Uschold-Schmidt N, Nyuyki KD, Fuchsl AM, Neumann ID \& Reber SO 2012 Chronic psychosocial stress results in sensitization of the HPA axis to acute heterotypic stressors despite a reduction of adrenal in vitro ACTH responsiveness. Psychoneuroendocrinology 37 1676-1687. (doi:10.1016/j. psyneuen.2012.02.015)

Ushijima K, Morikawa T, To H, Higuchi S \& Ohdo S 2006 Chronobiological disturbances with hyperthermia and hypercortisolism induced by chronic mild stress in rats. Behavioural Brain Research 173 326-330. (doi:10.1016/ j.bbr.2006.06.038)

Willner P 1990 Animal models of depression: an overview. Pharmacology \& Therapeutics 45 425-455. (doi:10.1016/0163-7258(90)90076-E)

Winkler D, Pjrek E, Praschak-Rieder N, Willeit M, Pezawas L, Konstantinidis A, Stastny J \& Kasper S 2005 Actigraphy in patients with seasonal affective disorder and healthy control subjects treated with light therapy. Biological Psychiatry 58 331-336. (doi:10.1016/j. biopsych.2005.01.031)

Yamazaki S, Numano R, Abe M, Hida A, Takahashi R, Ueda M, Block GD, Sakaki Y, Menaker M \& Tei H 2000 Resetting central and peripheral circadian oscillators in transgenic rats. Science 288 682-685. (doi:10.1126/science.288.5466.682)
Yamazaki S, Yoshikawa T, Biscoe EW, Numano R, Gallaspy LM, Soulsby S, Papadimas E, Pezuk P, Doyle SE, Tei H et al. 2009 Ontogeny of circadian organization in the rat. Journal of Biological Rhythms 24 55-63. (doi:10.1177/ 0748730408328438)

Yous S, Andrieux J, Howell HE, Morgan PJ, Renard P, Pfeiffer B, Lesieur D \& Guardiola-Lemaitre B 1992 Novel naphthalenic ligands with high affinity for the melatonin receptor. Journal of Medicinal Chemistry 35 1484-1486. (doi:10.1021/jm00086a018)

Zelena D, Mergl Z, Foldes A, Kovacs KJ, Toth Z \& Makara GB 2003 Role of hypothalamic inputs in maintaining pituitary-adrenal responsiveness in repeated restraint. American Journal of Physiology. Endocrinology and Metabolism 285 E1110-E1117.

Zohar J \& Westenberg HG 2000 Anxiety disorders: a review of tricyclic antidepressants and selective serotonin reuptake inhibitors. Acta Psychiatrica Scandinavica 403 39-49. (doi:10.1111/j.1600-0447.2000.tb10947.x)

Received in final form 20 August 2012

Accepted 20 September 2012

Made available online as an Accepted Preprint 20 September 2012 